\title{
Acute phase protein response in pigs experimentally co-infected with swine influenza virus and Bordetella bronchiseptica
}

\author{
MAEGORZATA POMORSKA-MÓL, IWONA MARKOWSKA-DANIEL, KRZYSZTOF KWIT
}

Department of Swine Diseases, National Veterinary Research Institute, Pulawy, Poland

\begin{abstract}
The response of four positive acute phase proteins $(A P P)$ was evaluated in pigs after co-infection with swine influenza virus (SIV) and Bordetella bronchiseptica (Bbr). Twelve piglets from a herd with high health status were used. Eight of them were infected intranasally with SIV and Bbr. The standard bacteriological methods for detection of Bbr and PCR tests for identification of Bbr and SIV in nasal swabs, lungs and bronchoalveolar fluids were used. serum APP concentrations were measured using commercial ELISA tests. Various kinetics of response was identified within the APP tested. $C$-reactive protein $(C R P)$, serum amyloid $A(S A A)$ and pig major acute protein (Pig-MAP) were characterized as fast and transient responders, while haptoglobin $(\mathrm{Hp})$ as a fast but very prolonged responder. The significant correlation was found between concentrations of $H p$ and $S A A$ in the serum and degree of turbinate atrophy as well as changes observed in the lungs. On the basis of our investigation we state that evaluation of $H p$ and SAA concentrations in the serum of pig co-infected with SIV and Bbr may provide useful information about severity of changes observed in the turbinates and lungs. Moreover, since all investigated APP strongly reacted after co-infection, it is clear that APP-measurements may reveal ongoing infection in pigs and could be the primary screening test prior to specific disease diagnosis. Future studies should focus on the possibility of distinguishing infected and non-infected pigs under field conditions.
\end{abstract}

Key words: pigs, acute phase protein response, SIV, Bordetella bronchiseptica.

(Centr Eur J Immunol 2012; 37 (3): 221-226)

\section{Introduction}

The acute phase protein (APP) response to inflammation or infection has been identified in a number of species and consists of alterations to the serum concentrations of several proteins, including APP [1-3]. Occurrence and monitoring progression of infection by APP measurements in blood is used extensively in human $[4,5]$. Similar diagnostic value of APP has been proposed in veterinary medicine. Moreover, APP are possible candidates to monitor the health status of pig herds [6].

Whilst APP responses have been observed for a large range of infections [1-3, 7-9] whether co-infection of swine influenza virus (SIV) H1N1 subtype with Bordetella bronchiseptica (Bbr) also induces an APPs response is not known. Co-infection with two or more pathogens is a common occurrence in respiratory diseases of most species, including pigs. Swine influenza virus is the important disease causing agents in pigs, not only as a primary pathogen but as a predisposing agent to secondary bacterial infection. Bordetella bronchiseptica is one of the etiologic agents causing atrophic rhinitis and pneumonia in swine and has been shown to contribute to the porcine respiratory disease complex (PRDC), a multifactorial complex of respiratory pathogens [10]. As it has been shown previously, SIV may enhance Bbr colonization in the respiratory tract [11].

The aim of this study was to assess whether experimentally induces co-infection, caused by SIV H1N1 subtype and Bbr, evokes an C-reactive protein (CRP), haptoglobin (Hp), serum amyloid A (SAA) or/and pig major acute phase protein (Pig-MAP) responses in pigs and whether the correlation between concentrations of investigated APPs in serum and pathological changes observed in infected pigs existed. To our knowledge there were no reports regarding APP response to SIV and Bbr co-infection in pigs.

Correspondence: Małgorzata Pomorska-Mól, Department of Swine Diseases, National Veterinary Research Institute, Al. Partyzantów 57, 24-100 Puławy, Poland, tel. +48 8188934 76, e-mail: mpomorska@piwet.pulawy.pl 


\section{Material and methods}

\section{Animals}

Twelve 6 weeks old piglets, both sexes, from a herd with very good health status were used. The herd was seronegative to porcine reproductive and respiratory syndrome virus and Aujeszky's disease virus. On the grounds of clinical, serological and pathological examinations no evidence of pleuropneumonia, streptococcosis or atrophic rhinitis was recorded at any age group of pigs on the farm. During the experiment, piglets were housed at the BSL3 animal facility of the National Veterinary Research Institute (Puławy, Poland) in two independent units, one for the control and one for the infected pigs. Animal use and handling protocols were approved by Local Ethical Commission (University of Life Sciences in Lublin, Poland).

\section{Influenza virus and Bordetella bronchiseptica inocula}

Swine influenza virus A/sw/Poland/KPR9/2004 (H1N1 subtype) (hereafter referred to as SwH1N1), which had been isolated from the lungs of fattening pig with swine influenza in 2004, was used for the experimental infection. The stock used for inoculation represented the third passage in eggs. The virus concentration was evaluated in Madin-Darby canine kidney (MDCK) cells and stored at $-80^{\circ} \mathrm{C}$ until used. Inoculations of $10^{7,3} \mathrm{TCID}_{50}$ of virus in $2 \mathrm{ml}$ of PBS were given.

Twenty four hours culture of Bbr field strain, isolated from a pig with clinical form of non-progresive athrophic rhinitis (NPAR), suspended in a phosphate-buffered saline (PBS) to a final concentration of $1.7 \times 10^{8} \mathrm{cfu} / \mathrm{ml}$ was used for inoculation $(2 \mathrm{ml} / \mathrm{pig})$.

The bacterial and viral inoculums were prepared and delivered separately to both nostrils.

\section{Experimental design}

On day 0 , eight piglets were inoculated intranasally with SwH1N1 and Bbr. Four mock-inoculated pigs (with PBS) served as control pigs.

In order to examine the events taking place at the early stages of infection with SwH1N1 three infected and one control piglets were euthanized on days 3 after infection Remaining pigs from each group (5 infected and 3 control) were euthanized on 10 dpi. Necropsy was performed immediately after the animals were euthanized.

\section{Clinical and pathological examination}

Rectal temperatures were assessed daily and clinical signs of disease were recorded. Fever was defined as body temperature of $>40^{\circ} \mathrm{C}$.

Blood samples were collected on days $-7,0$ (challenge), 1, 2, 3, 5, 7 and 10 dpi. Serum was harvested after centrifugation and stored at $-20^{\circ} \mathrm{C}$ for further analyses.
Nasal swabs were taken at 2, 3, 4, 5 and 10 dpi. Complete necropsy was done on each animal, with special emphasis on the respiratory tract. Samples from lungs, tracheas and BALF were collected aseptically for further analysis.

\section{Turbinate score}

The snouts were sectioned at the upper first premolar tooth during necropsy. The lesions in the left and right turbinates and septum were scored as $0,1,2$, and 3, as was described previously [12]. Normal turbinates were graded as 0 . Slight but obvious atrophy was graded as 1 . Moderate atrophy of not less than half of the turbinates was graded at 2. Severe atrophy of the dorsal and ventral scrolls was graded as 3 . The three scores (from left and right turbinates and septum) were then added together and divided by 3 , to determine final visual turbinate scores (TS) for each piglet, ranging from 0 to 3 .

\section{Lung score}

Lungs were assessed according to the scheme described by Christensen et al. [13]. If no changes were found in the lobe, it was scored as $0 \%$. Changes in the right lung were scored as follows: apical lobe $10 \%$, cardiac lobe $10 \%$, diaphragmatic lobe $35 \%$ (all together 55\%). Changes observed in the left lung were scored as: apical lobe 5\%, cardiac lobe $5 \%$, diaphragmatic lobe $30 \%$. Changes in intermediate lobe was scored as 5\%. All recorded scores were then added together, to determine final visual lung score (LS) for each pig, ranging from 0 to $100 \%$.

\section{Laboratory examination}

\section{Swabs and tissue samples}

Nasal swabs, lung samples, and BALF were tested for the presence of Bbr using standard bacteriological methods as previously described [14]. Additionally, the PCR technique was performed for both, clinical samples and cultures. Strains of Bbr were identified by amplification of the dermonecrotoxin (DNT) gene according to procedure described by Stępniewska and Markowska-Daniel [15].

For detection of SIV the general swine influenza A real time reverse transcription (RRT)-polymerase chain reaction (PCR) method (the "perfect match" M gene RRT PCR), as described previously [16], was used. Samples with Ct value $<30$ were considered to be $\mathrm{M}$ gene positive, samples with $\mathrm{Ct}$ value 30-35 with sigmoidal/logarithmic appearance were considered to be weak positive, samples with $\mathrm{Ct}$ value $>35$ were considered to be negative.

\section{Measurement of acute phase proteins}

For determination of APP commercial ELISAs were used according to the manufacturer's recommendation (Pig C-reactive protein and Pig haptoglobin from Life Diagnostics Inc., USA; PigMAP KIT ELISA from PigCHAMP Pro Europa S.A, Spain; Phase Serum Amyloid A Assay from Tri- 

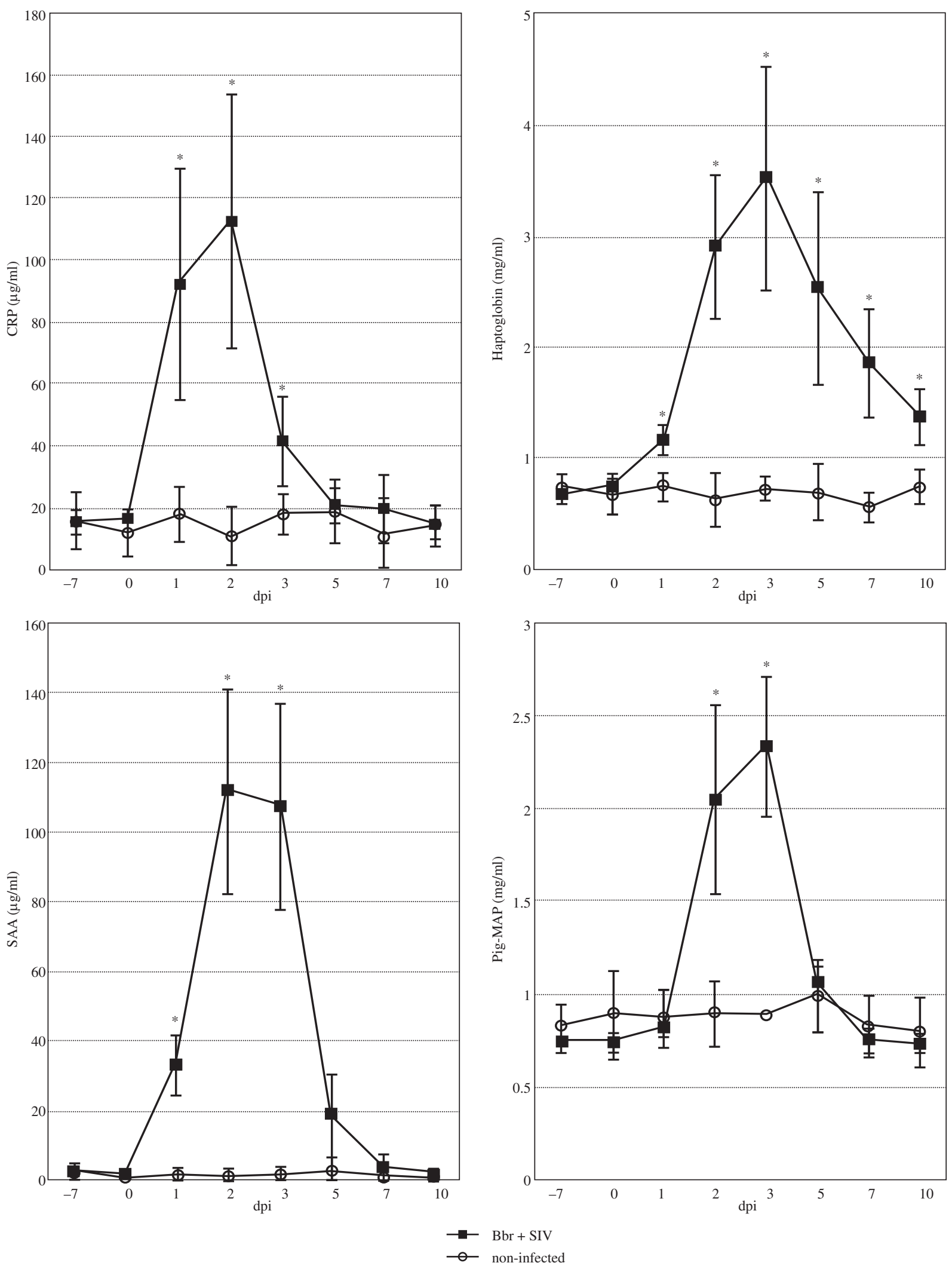

Fig. 1. Concentrations of CRP, haptoglobin, SAA and Pig-MAP in serum in control pigs and pigs infected with SIV and $B$. bronchiseptica. Data are geometric means $\pm \mathrm{SD}$; dpi - days post inoculation. $* P<0.05$ as compared to the control animals 
delta Development Ltd, Ireland). For all analyses serum samples were tested in duplicate. Prior to analyses serum samples were diluted as follows: $1: 1000$ for CRP, $1: 35000$ for Hp, $1: 500$ for SAA and $1: 1000$ for Pig-MAP.

\section{Statistical analysis}

A Kruskal-Wallis test with post hoc multiple comparisons for comparison of all pairs was used for comparison of mean APP concentrations. For analysis of correlation between measured parameters, the Spearman-Rang correlation was used. Differences with $\alpha<0.05$ were considered as significant. All calculations were performed with the Statistica 8.0 (Statsoft, Poland) computer program.

\section{Results}

\section{Clinical signs}

Infection method used in present study induced clinical sings, including sneezing and coughing in all infected pigs. In four out of eight piglets also accelerated respiratory rates and dyspnoea were observed. The body temperature of inoculated pigs increased over $40^{\circ} \mathrm{C}$, from 2 to $5 \mathrm{dpi}$. In the control piglets no clinical sings of any disease were seen.

\section{Microbiological and pathological examination}

No Bbr were found (bacteriological examination and PCR test) in the nasal swabs taken before inoculation. Reisolation of Bbr from nasal swabs was successful only in 4, while from BALF in 5 inoculated piglets. The use of PCR technique improved detection of Bbr from nasal swabs (8/8) and from lungs (7/8) and BALF (6/8).

$\mathrm{M}$ gene RRT-PCR assay, used to confirm the presence of SIV in the nasal swabs revealed positive results from all infected pigs between 2 and 5 dpi. In the nasal swabs taken before inoculation, and these from control pigs, no SIV genetic material was found. No viral RNA was detected in swabs at 10 dpi. The presence of SIV RNA was also analyzed in tracheas and lungs. In all infected pigs, euthanized at $3 \mathrm{dpi}$, the presence of SIV were confirmed for trachea as well as lungs. No viral RNA was detected in lungs on day 10 post inoculation.

The slight atrophy of turbinates was observed in 4 out of 5 inoculated piglets euthanized at $10 \mathrm{dpi}($ mean TS $=0.66$ \pm 0.4 , range $0-1.33$ ).

Postmortem examination revealed macroscopic lesions in the lungs of $8 / 8$ infected pigs. The mean lung score was $22 \% \pm 12.55 \%$ (range $10-35 \%$ ).

\section{Acute phase proteins}

All APP were strongly induced after inoculation. The concentrations of APP in infected piglets revealed significant changes during the study period (Fig. 1). In the control piglets levels of all investigated APP remained relatively constant.
Prior to inoculation all piglets had CRP-values below $20.1 \mu \mathrm{g} / \mathrm{ml}$ (mean $15.76 \pm 3.96$ ). Twenty four hours after inoculation, the mean CRP level was significantly higher (over 6-fold increase) as compared to day 0 concentration $(p<0.05)$. The mean maximum concentration of CRP was observed at 2 dpi (almost 8-fold increase). Starting from 5 dpi after inoculation, the mean concentration of CRP did not differ significantly from that observed on day 0 , as well that observed in the control piglets $(p \geq 0.05)$.

Basal serum concentration of Hp ranged from 0.56 to $0.87 \mathrm{mg} / \mathrm{ml}$. Changes in Hp concentrations were observed in all infected pigs. Significant increase in the Hp concentration was observed as early as in $1 \mathrm{dpi}(p<0.05)$. The mean peaked level was almost 5 -fold higher compared to mean pre-inoculation concentration. The highest individual concentrations of $\mathrm{Hp}$ were observed between the second and third dpi. The levels of Hp remain significantly elevated to the end of study $(p<0.05)$.

The mean concentration of SAA significantly increase $24 \mathrm{~h}$ post inoculation. The statistically significant increase in mean SAA concentration, as compared to the control piglets, were observed on days 1, 2, and 3 after infection $(p<0.05)$. The highest concentrations of SAA in particular animals were seen between 2 and 3 dpi. Mean peak level reached 112.08 $\pm 29.65 \mu \mathrm{g} / \mathrm{ml}$ and was over 40 -fold higher compared to day 0 -level. From day 5, the SAA concentration reached concentration similar as in control pigs $(p>0.05)$.

Preinoculation levels of Pig-MAP were found to be below $0.85 \mathrm{mg} / \mathrm{ml}$ (mean $0.74 \pm 0.05 \mathrm{mg} / \mathrm{ml}$ ). Concentration of Pig-MAP significantly increased at $2 \mathrm{dpi}(p<0.05)$ and remained significantly elevated to $3 \mathrm{dpi}$. The maximum mean concentration of Pig-MAP, observed at $3 \mathrm{dpi}$, was over 3 times higher as compared to day 0 . The mean concentration of Pig-MAP returned to preinoculation value at 5 dpi.

A strong correlations were found between concentrations of Hp and SAA in serum and changes in the lungs in inoculated piglets ( $r=0.79$ and 0.71 , respectively) as well as concentrations of these proteins and changes in the turbinates ( $r=0.83$ and 0.78 , respectively). All correlations were significant $(p<0.05)$.

\section{Discussion}

The previously described potential use of APP [1-3, $7,9]$ prompted us to look at the course of the main APP serum concentrations after co-infection of pigs with SIV and $\mathrm{Bbr}-$ an important pathogens of swine. Although swine influenza is typically a self-limited infection characterized by high-morbidity and low mortality, secondary infections can significantly increases mortality. Bacterial pneumonia secondary to SIV infection is often observed in pigs, as SIV is a key contributor to the PRDC. Bacterial pathogen associated with PRDC include i.a. Bordatella bronchiseptica, an ubiquitous pathogen of pigs. 
Previous studies in pigs have described the kinetics of APP response in the course of various diseases [1, 3, 8, 17]. Up till now, though, there have not been any reports published on the development and dynamics of the of acute phase response after co-infection of pigs with SIV and Bbr, a situation very common in the field conditions.

Therefore, in our study, an early response of CRP, Hp, SAA and Pig-MAP during experimentally induced co-infection was characterized. In addition, the relationship between APP concentrations and pathological changes was analyzed.

CRP was induced from a level of around $16 \mu \mathrm{g} / \mathrm{ml}$ to about $112 \mu \mathrm{g} / \mathrm{ml}$. Similar increases were found by Sorensen et al. [9] after Streptococcus suis (S. suis) inoculation and by Lampreave et al. [18] upon turpentine induced inflammation. The very fast, non-protracted response of CRP in our study was in accordance with results of previous experiments in pigs $[9,18]$.

Almost a 5-fold increase in Hp, after co-infection, places pig Hp into the category of moderate APP [6]. Similar induction levels and relative increases in pig Hp were observed by others [9, 17] during monoethiological infections.

Similar increase in Pig-MAP, with comparable absolute levels was found by Grau-Roma et al. [7] in pigs with post-weaning multisystemic wasting syndrome. On the other hand, Heegaard et al. [2] found over a 13-fold increase in Pig-MAP after experimental infection with A. pleuropneumoniae. The kinetics of Pig-MAP response after inoculation with S. suis [9] was generally similar to but more protracted than the response seen in the presented study. In accordance with data presented by Heegaard et al. [2] the level of Pig-MAP increased later than the levels of Hp and CRP.

In agreement with previous reports [2, 9], SAA was clearly found to be a major APP in pig. Its mean induction level was over 40-fold higher than before inoculation. A similar strong induction of SAA in pigs was observed after S. suis infection [9].

Summarizing, after co-infection of piglets with SIV and $\mathrm{Bbr}$, various kinetics of responses could be identified within the APP tested. CRP, SAA and Pig-MAP could be characterized as fast and transient responders, while $\mathrm{Hp}$ as a fast and very prolonged responders.

In present study the significant correlation was found between concentrations of $\mathrm{Hp}$ and SAA in the serum and degree of turbinate atrophy, what is in agreement with results obtained by Francisco et al. [19], who found a slight correlation between $\mathrm{Hp}$ concentration in serum and extent of turbinate atrophy after infection of pigs with Bbr. Similarly, as in previous studies in pigs, strong correlations were also observed between concentration of $\mathrm{Hp}$ or SAA and changes observed in the lungs [3].

Data presented in our paper provide clear evidence that all investigated APP strongly reacted after co-infection, thus, APP-measurements may reveal ongoing co-infection and could be the primary screening test prior to specific disease diagnosis. Additionally, on the basis of our investigation we can state that evaluation of $\mathrm{Hp}$ and SAA concentrations in the serum of pig co-infected with SIV and Bbr may provide useful information about severity of changes observed in the turbinates and lungs and in this way can help to evaluate the severity of infection. With the above information in mind the better treatment can be applied. Moreover, monitoring of APP concentrations in serum may be useful for selecting clinically healthy pigs before integration into an uninfected herd. Future studies should focus on the determination of references values for various APP and possibility of distinguishing infected and non-infected pigs under field conditions.

This work was supported by Project N N308 235938 founded by the Ministry of Science and Higher Education.

\section{References}

1. Carpintero R, Alonso C, Pineiro M, et al. (2007): Pig major acute phase protein and apolipoprotein A-I responses correlate with clinical course of experimentally induced African swine fever and Aujeszky's disease. Vet Res 38: 741-753.

2. de la Fuente MAJ, Carpineiro R, Rodriguez-Ferri EF, et al. (2008): Acute-phase response in pigs experimentally infected with Haemophilus parasuis. Comp Immunol Microbiol Infec Dis 33: 455-465.

3. Pomorska-Mól M, Markowska-Daniel I, Kwit K, et al. (2011): Kinetics of the response of four positive acute phase proteins in pigs experimentally infected with toxigenic Pasteurella multocida. Vet Microb 152: 429-435.

4. Chai LA, Netea MG, Teerenstra S, et al. (2010): Early proinflammatory cytokines and C-reactive protein trends as predictors of outcome in invasive aspergillosis. J Infect Dis 202: 1454-1462.

5. Tsiakalos A, Karatzaferis A, Ziakas P, Hatzis G (2009): Acutephase proteins as indicators of bacterial infection in patients with cirrhosis. Liver Int 29: 1538-1542.

6. Murata H, Shimada N, Yoshioka M (2004): Current research on acute phase proteins in veterinary diagnosis: an overview. Vet J 168: 28-40.

7. Grau-Roma L, Heegaard PMH, Hjulsager CK, et al. (2009): Pig-major acute phase protein and haptoglobin serum concentrations correlate with PCV2 viremia and the clinical course of postweaning multisystemic wasting syndrome. Vet Microbiol 138: 53-61.

8. Heegaard PMH, Klausen J, Nielsen JP, et al. (1998): The porcine acute phase response to infection with Actinobacillus pleuropneumoniae. Haptoglobin, C-reactive protein, major acute phase protein and Serum amyloid A protein are sensitive indicators of infection. Comp Biochem Physiol 119B: 365-373.

9. Sorensen NS, Tegtmeier C, Andresen LO, et al. (2006): The porcine acute phase protein response to acute clinical and subclinical experimental infection with Streptococcus suis. Vet Immunol Immunopathol 113: 157-168.

10. Brockmeier SL, Register KB, Magyar T, et al. (2002): Role of the dermonecrotic toxin of Bordetella bronchiseptica in the pathogenesis of respiratory disease in swine. Infec Immun 2: 481-490.

11. Loving CL, Brockmeier SL, Vincent AL, et al. (2010): Influenza virus coinfection with Bordetella bronchiseptica enhances 
bacterial colonization and host responses exacerbating pulmonary lesions. Microb Pathogenesis 49: 237-245,

12. Gatlin CL, Jordan WH, Shryock TR, Smith WC (1996): The quantitation of turbinate atrophy in pigs to measure the severity of induced atrophic rhinitis. Can J Vet Res 60: 121-126,

13. Christensen G, Sorensen V, Mousing J (1999): Diseases of the respiratory system. In: Diseases of swine. Straw BE, D’Allaire S, Mengeling WL, Taylor DJ, Ames, USA, 1999; 925-929.

14. Markowska-Daniel I, Stępniewska K, Pejsak Z (2010): Detection of Pasteurella multocida and Bordetella bronchiseptica, etiological agents of atrophic rhinitis, by means of PCR tests. Medycyna Wet 66: 45-50.

15. Stepniewska K, Markowska-Daniel I (2010): Evaluation of PCR test for detection of dermonecrotoxin of Bordetella bronchiseptica. Bull Vet Inst Pulawy 54: 495-499.

16. Slomka MJ, Densham AL, Coward VJ, et al. (2010): Real time reverse transcription (RRT)-polymerase chain reaction (PCR) methods for detection of pandemic (H1N1) 2009 influenza virus and European swine influenza A virus infections in pigs. Influenza Other Respi Viruses 4: 277-293.

17. Hall WF, Eurell TE, Hansen RD, Herr LG (1992): Serum haptoglobin concentration in swine naturally or experimentally infected with Actinobacillus pleuropneumoniae. J Am Vet Med Assoc 201: 1730-1733.

18. Lampreave F, González-Ramón N, Martínez-Ayensa S, et al. (1994): Characterization of the acute phase serum protein response in pigs. Electrophoresis 15: 672-676.

19. Francisco CJ, Shryock TR, Bane DP, Unverzagt L (1996): Serum haptoglobin concentration in growing swine after intranasal challenge with Bordatella bronchiseptica and toxigenic Pasteurella multocida type D. Can J Vet Res 60: 222227. 